\title{
Comparison of 3D intraoperative digital subtraction angiography and intraoperative indocyanine green video angiography during intracranial aneurysm surgery
}

\author{
*Serge Marbacher, MD, PhD, Itai Mendelowitsch, MD," Basil Erwin Grüter, MD,1 \\ Michael Diepers, MD, ${ }^{2}$ Luca Remonda, MD, ${ }^{2}$ and Javier Fandino, MD' \\ 1Department of Neurosurgery and 'Division of Neuroradiology, Department of Radiology, Kantonsspital Aarau, Switzerland
}

\begin{abstract}
OBJECTIVE During the last decade, improvements in real-time, high-resolution imaging of surgically exposed cerebral vasculature have been realized with the successful introduction of intraoperative indocyanine green video angiography (ICGVA) and technical advances in intraoperative digital subtraction angiography (DSA). With the availability of 3D intraoperative DSA (3D-iDSA) in hybrid operating rooms, the present study offers a contemporary comparison for rates of accuracy and discordance.
\end{abstract}

METHODS In this retrospective study of prospectively collected data, 140 consecutive patients underwent microsurgical treatment of intracranial aneurysms (IAs) in a hybrid operating room. Variables analyzed included patient demographics, aneurysm-specific characteristics, intraoperative ICGVA and 3D-iDSA findings, and the need for intraoperative clip readjustment. The authors defined the discordance rate of the two modalities as a false-negative finding that necessitated clip repositioning after 3D-iDSA.

RESULTS In 120 patients, ICGVA and 3D-iDSA were used to evaluate 134 IA obliterations. Of 215 clips used, 29 (14\%) were repositioned intraoperatively, improving the surgical result in all 29 patients (24\%). Repositioning was prompted by visual inspection and microvascular Doppler ultrasonography in 8 (28\%), ICGVA in $13(45 \%)$, and 3D-iDSA in 7 (24\%) patients. Clip repositioning was needed in 7 patients (6\%) based on 3D-iDSA, yielding an ICGVA accuracy rate of $94 \%$. Five $(71 \%)$ of the ICGVA-3D-iDSA discordances that prompted clip repositioning occurred at the anterior communicating artery complex.

CONCLUSIONS A combination of vascular monitoring techniques most often achieved correct intraoperative interpretation of complete IA occlusion and parent artery integrity. Compared with 3D-iDSA imaging, ICGVA demonstrated high accuracy. Despite the relatively low discordance rate, iDSA was confirmed to be the gold standard. Improved imaging quality, including 3D-iDSA, supports its routine use in IA surgery, obviating the need for postoperative DSA.

https://thejns.org/doi/abs/10.3171/2018.1.JNS172253

KEYWORDS intraoperative; indocyanine green video angiography; three-dimensional digital subtraction angiography; clipping; hybrid operating room; vascular disorders

$\mathrm{S}$ URGICAL treatment of intracranial aneurysms (IAs) requires a careful balance between the most complete aneurysm occlusion and preservation of parent arteries and perforators. Since Allcock and Drake's ${ }^{3} 1963$ publication that showed high rates of rebleeding from IA remnants after surgery and that advocated routine postoperative angiography, subsequent series have reported ranges of IA remnants from $1 \%$ to $36 \%$ and vessel occlusion from $1 \%$ to $25 \%$. $^{3,12,13,21,37}$ Given the relatively high rate of these adverse findings, Grossart and Turner's ${ }^{17} 1974$ study recommended the use of intraoperative angiography to immediately verify the surgical results and alter the therapy if needed. Despite proven effectiveness $(6 \%-35 \%$ of relevant information), intraoperative digital subtraction angiography (iDSA) demonstrates relatively high rates of false-negative findings (range 0\%-13\%) compared with the highest-quality postoperative DSA.5,22,28,30,39

In 2003, Raabe et al. ${ }^{32}$ introduced indocyanine green

ABBREVIATIONS ACA = anterior cerebral artery; $\mathrm{ACOA}=$ anterior communicating artery; $\mathrm{CTA}=\mathrm{CT}$ angiography; $\mathrm{DSA}=$ digital subtraction angiography; $\mathrm{IA}=$ intracranial aneurysm; ICGVA = indocyanine green video angiography; iDSA =intraoperative DSA; PCOA = posterior communicating artery.

SUBMITTED October 1, 2017. ACCEPTED January 16, 2018.

INCLUDE WHEN CITING Published online July 13, 2018; DOI: 10.3171/2018.1.JNS172253.

* S.M. and I.M. share first authorship of this work. 
video angiography (ICGVA) as a new method to intraoperatively assess vascular flow, reporting comparable efficiency (9\% significant findings) and accuracy (90\% correspondence) with iDSA. ${ }^{33}$ Subsequently, the few published comparisons confirmed ICGVA and iDSA rates of effectiveness (3\%-8\%) and accuracy (86\%-98\%). ${ }^{4,10,18,43} \mathrm{~A}$ number of other studies noted the utility of ICGVA in contributing 2\%-38\% of significant findings and reported discordance rates with postoperative DSA that ranged from $4 \%$ to $14 \% .{ }^{19,24,27,29,35,36,41}$ These figures are comparable with the rates of false-negative findings between intraoperative and postoperative DSA. Although DSA is considered the gold standard for intraoperative evaluation, its high falsenegative rates prevented its adoption as a standard of care that obviates the need for postoperative DSA.

With significant advances in imaging quality for iDSA and the availability of 3D-iDSA in hybrid operating rooms, our study offers a contemporary comparison of vascular imaging techniques. Compared with conventional DSA, cumulative evidence suggests that 3D-DSA yields more detailed information of the angioarchitecture and significant benefits for evaluation of clipped IAs. $2,20,23,38,40,44$ In this retrospective study of patients treated for IAs, we evaluate the accuracy of routine use of 3D-iDSA and ICGVA and rates of discordance during a 4-year period.

\section{Methods}

We retrospectively reviewed our prospectively collected data for 140 consecutive patients who underwent craniotomy and IA clipping in our hybrid operating room between November 2011 and February 2015. Nineteen patients were excluded because they had visual inspection, Doppler ultrasonography, and 3D-iDSA alone without ICGVA; another patient was excluded because he had undergone intraoperative wrapping alone.

Baseline characteristics included patient age, sex, rupture status, Fisher grade (if the lesion was ruptured), IA occlusion technique, number of clips per aneurysm, and IA location and size. We reviewed operative records and videos (including ICGVA) to identify any clip that was repositioned based on visual inspection and/or microvascular Doppler ultrasonography; relevant surgical information provided by ICGVA; and consistency of 3D-iDSA findings with postclipping ICGVA findings and need for surgical intervention. False-positive and false-negative findings without the need for surgical intervention were not recorded. The study was approved by our institutional review board and the Swiss ethics commission.

\section{Clinical Protocol}

After admission for elective surgery to the hospital or to our emergency department, patients treated for IAs routinely underwent preoperative assessment by $\mathrm{CT}$ angiography (CTA) and 3D-DSA. An interdisciplinary team discussed each case to determine if surgical, endovascular, or both interventions were required based on IA configuration, location, the patient's condition, and resources. ${ }^{26}$

After exposing the IA angioarchitecture, parent and branching arteries were assessed using microvascular
Doppler ultrasonography (probe $16 \mathrm{MHz}$, power $6 \mathrm{~mW}$; LOOKI, Atys Medical) before and after clip ligation. If clip position was deemed satisfactory, ICGVA was performed for at least 1 minute; the video analysis was then correlated with the findings from visual inspection and parent artery Doppler ultrasonography. If the IA was correctly occluded, 3D-iDSA was performed. If the clip needed adjustment, the protocol flow was repeated. Before wound closure, all patients underwent intraoperative CT to rule out spaceoccupying hematoma formation.

\section{Indocyanine Green Video Angiography}

Detailed technical aspects of ICGVA application have been described previously. ${ }^{32,33}$ Flow in arteries, branching vessels, and perforators was observed in real time and on video loops. The flow pattern and the aneurysm itself were monitored for at least 1 minute to diagnose potential late filling of the clipped IA. If ICGVA was repeated, dye injection was delayed for 5 minutes or more after the previous injection to reduce residual fluorescent activity.

\section{Intraoperative Digital Subtraction Angiography}

The equipment and setting of our hybrid operating room have been described previously. ${ }^{14}$ Briefly, the surgical carbon fiber table (Alphamaquet 1150, Maquet AG) was coupled with a C-arm angiography system (Allura Xper FD20, Philips Medical Systems). Use of radiolucent head holders with radiolucent head pins optimized the acquisition of angiograms and intraoperative CT scans (Mayfield, Integra $\mathrm{GmbH}$ ). At least 3 standard projections (anteroposterior, lateral, and oblique) and rotational angiography were performed by manual arterial injection of contrast agent (IOPAMIRO $300 \mathrm{mg} / \mathrm{ml}, 5-\mathrm{ml}$ bolus, Bracco Suisse). The volumetric sequence was used to reconstruct 3D angiographic and multiplanar CT data sets. After data sets were compared to preoperative images, the findings were discussed by the attending interventional neuroradiologist and neurosurgeon. There were no associated complications with iDSA.

\section{Statistical Analysis}

ICGVA and 3D-iDSA images were analyzed to detect significant findings (i.e., IA sac or neck remnants or hemodynamically relevant parent artery stenosis) that prompted additional clip placement or clip repositioning. A model based on a multivariate logistic regression of potential predictor variables (IA size and location; rupture status; Fisher grade; and number of clips applied) could not be established with only 7 events for the outcome variable of ICGVA-3D-iDSA discordance.

\section{Results}

Of 140 consecutive patients, the 120 patients who were studied included 39 men and 81 women who averaged 55 years of age (range 19-79 years); these patients underwent treatment for 134 IAs, including $73(55 \%)$ ruptured and $61(45 \%)$ unruptured aneurysms. Most aneurysms were small; specifically, $58 \%$ were $<7 \mathrm{~mm}$, and $92 \%$ occurred in the anterior circulation at the middle cerebral artery in 
TABLE 1. Clinical characteristics of ICGVA and 3D-iDSA discordance and concordance

\begin{tabular}{|c|c|c|c|}
\hline Variable & Total No. (\%) & Discordance & Concordance \\
\hline Patients & $120(100)$ & $7(5.8)$ & $113(94.2)$ \\
\hline IAs & $134(100)$ & $7(5.2)$ & $127(94.8)$ \\
\hline Clips & $215(100)$ & $15(7.0)$ & $200(93.0)$ \\
\hline Female & $81(67.5)$ & $6(85.7)$ & $75(66.4)$ \\
\hline Mean age (yrs) & 55 & 60 & 55 \\
\hline Ruptured IAs & $61(45.5)$ & $4(57.1)$ & $57(44.9)$ \\
\hline \multicolumn{4}{|l|}{ Fisher grade } \\
\hline 1 & $5(8.2)$ & $1(25)$ & $4(7)$ \\
\hline 2 & $11(18)$ & $1(25)$ & $10(17.5)$ \\
\hline 3 & $9(14.8)$ & $1(25)$ & $8(14)$ \\
\hline 4 & $36(59)$ & $1(25)$ & $35(61.5)$ \\
\hline \multicolumn{4}{|c|}{ Aneurysm size (mm) } \\
\hline$<7$ & $78(58.2)$ & $4(57.1)$ & $74(58.3)$ \\
\hline $7-12$ & $52(38.8)$ & $1(14.3)$ & $51(40.1)$ \\
\hline $13-24$ & $4(3.0)$ & $2(28.6)$ & $2(1.6)$ \\
\hline$>24$ & $0(0)$ & $0(0)$ & $0(0)$ \\
\hline \multicolumn{4}{|l|}{ Aneurysm location } \\
\hline MCA & $60(45.5)$ & $1(14.3)$ & $59(47.2)$ \\
\hline $\mathrm{ACoA}$ & $37(26.5)$ & $5(71.4)$ & $32(24)$ \\
\hline Proximal ACA & $13(9.8)$ & $0(0)$ & $13(10.4)$ \\
\hline ICA & $11(8.3)$ & $0(0)$ & $11(8.8)$ \\
\hline PCoA & $8(6.1)$ & $1(14.3)$ & $7(5.6)$ \\
\hline AChA & $2(1.5)$ & $0(0)$ & $2(1.6)$ \\
\hline $\mathrm{BA}$ & $3(2.3)$ & $0(0)$ & $3(2.4)$ \\
\hline
\end{tabular}

$\mathrm{AChA}=$ anterior choroidal artery; $\mathrm{BA}=$ basilar artery; $\mathrm{ICA}=$ internal carotid artery; $\mathrm{MCA}=$ middle cerebral artery; $\mathrm{PCOA}=$ posterior communicating artery.

$60(46 \%)$, anterior communicating artery (ACoA) complex in $37(27 \%)$, proximal anterior cerebral artery (ACA) in $13(10 \%)$, internal carotid artery in $11(8 \%)$, and anterior choroidal artery in $2(2 \%)$. Clinical characteristics stratified by ICGVA-3D-iDSA concordance and discordance are summarized (Table 1).

Of the 215 clips placed in 134 aneurysms, 29 (14\%) were repositioned intraoperatively. Of these 29 clips, malposition was detected in $8(7 \%)$ after visual inspection and microvascular Doppler ultrasonography, in $13(11 \%)$ after ICGVA, in $7(6 \%)$ after visualization of IA remnants or parent artery compromise by intraoperative 3D-DSA, and in $1(1 \%)$ after unexpected intraoperative rupture (Fig. 1). The significant findings detected on 3D-iDSA not visible after ICGVA in 7 patients resulted in a discordance rate of $6 \%$ (7/120). Considering all the intraoperative tools used to inspect the completeness of IA occlusion and concomitant integrity of parent arteries, the 29 clips repositioned thus improved the operative safety for all 29 , or $24 \%$ of patients (Table 2 and illustrative cases in Fig. 2).

Of the 7 IAs with ICGVA-3D-iDSA discordances, 5 were small $(\leq 7 \mathrm{~mm})$ and 5 were located at the ACoA complex. The 3D-iDSA revealed a significant residual aneurysm in 5 patients and relevant parent artery occlusion in 2 patients (Table 2 ).

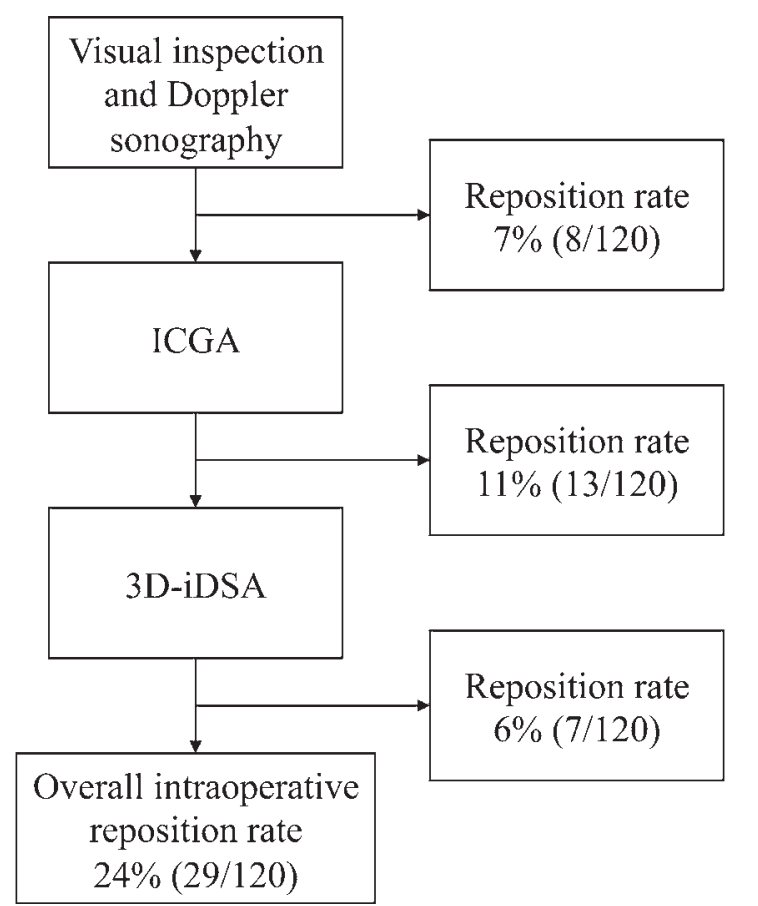

FIG. 1. Flowchart of intraoperative clip reposition rate according to various modalities for assessment of aneurysm occlusion and parent artery flow. ICGA = indocyanine green video angiography.

\section{Illustrative Cases}

\section{Case 1: Aneurysm Remnant Treated by Additional Clip}

This 61-year-old patient presented with an unruptured 7-mm bilobular ACoA aneurysm (Fig. 2A1). After single clip placement and visual control, Doppler ultrasonography showed no residual blood flow in the aneurysm sac and patent parent arteries. ICGVA confirmed complete occlusion and prompt filling of the ipsilateral and contralateral $\mathrm{A}_{2}$ segments. However, 3D-iDSA revealed a residual aneurysm remnant of the more posteriorly projecting part of the bilobular aneurysm beneath the tips of the clip blades (Fig. 2A2). After applying an additional curved clip, repeated 3D-iDSA demonstrated complete obliteration without any residual aneurysm neck (Fig. 2A3).

\section{Case 2: Aneurysm Remnant Treated by Wrapping}

This 68-year-old patient was admitted with SAH caused by a ruptured 6-mm ACoA aneurysm (Fig. 2B1). Visual inspection and microvascular Doppler ultrasonography demonstrated complete aneurysm occlusion and integrity of both $\mathrm{A}_{2}$ segments. Inspection by ICGVA showed no residual aneurysm filling and patency of parent arteries. Only 3D-iDSA revealed a slight bulging beneath the middle portion of the clip (Fig. 2B2). Since the surgeon judged that repositioning of the clip was unlikely to improve the surgical result, the aneurysm remnant was circumferentially wrapped with temporalis muscle (Fig. 2B3).

\section{Case 3: Aneurysm Remnant Treated by Clip Repositioning}

This 60-year-old patient underwent clip ligation of a small 6-mm ACoA aneurysm (Fig. 2C1). Multiple clips 
TABLE 2. Clinical characteristics of ICGVA and 3D-iDSA discordance and concordance

\begin{tabular}{|c|c|c|c|c|c|c|c|}
\hline Case No. & SAH & Fisher Grade & Aneurysm Location & Aneurysm Size (mm) & No. of Clips & 3D-iDSA Findings & Surgical Intervention \\
\hline 1 & No & & $\mathrm{ACoA}$ & 7 & 2 & IA remnant & Additional clip \\
\hline 2 & Yes & 3 & $\mathrm{ACoA}$ & 6 & 1 & IA remnant & Additional wrapping \\
\hline 3 & Yes & 4 & $\mathrm{ACoA}$ & 3 & 2 & IA remnant & Clip repositioning \\
\hline 4 & Yes & 2 & PCoA & 5 & 2 & Parent artery compromise & Clip repositioning \\
\hline 5 & No & & $\mathrm{ACoA}$ & 15 & 2 & IA remnant & Clip repositioning \\
\hline 6 & No & & MCA & 14 & 2 & Parent artery compromise & Clip repositioning \\
\hline 7 & Yes & 1 & $\mathrm{ACoA}$ & 6 & 3 & IA remnant & Clip repositioning \\
\hline
\end{tabular}

$\mathrm{SAH}=$ subarachnoid hemorrhage.

were applied to secure the fundus of the ruptured aneurysm. Visual inspection and ICGVA assessment of the aneurysm sac after clip application was not feasible because of the restricted angle of the surgical exposure. 3D-iDSA demonstrated significant residual aneurysm filling in the extension of the aneurysm tips (Fig. 2C2). Clips were removed one by one and repositioned more deeply. Control 3D-iDSA revealed complete occlusion of the aneurysm (Fig. 2C3).

\section{Case 4: Parent Vessel Impairment Resolved by Clip Repositioning}

In this 54-year-old patient, preoperative four-vessel DSA showed a large fetal posterior communicating artery ( $\mathrm{PCoA}$ ) variant as the primary supply to the posterior cerebral artery. The neck of the ruptured aneurysm originated solely off the PCoA itself, a few millimeters off the internal carotid artery (Fig. 2D1). Although visual inspection, Doppler ultrasonography, and ICGVA demonstrated complete aneurysm occlusion, assessment of the parent artery was limited within the deep operative field of this true PCoA aneurysm. Hemodynamically relevant stenosis was detected by 3D-iDSA (Fig. 2D2). Clip repositioning resulted in preservation of the parent artery (Fig. 2D3).

\section{Discussion}

In our retrospective review of 120 patients with IAs who underwent clipping in a hybrid operating room, comparable accuracy was afforded by routine use of 3D-iDSA and ICGVA to assess the completeness of IA occlusion and preservation of parent artery patency. Despite the overall low $6 \%$ discordance rate that then necessitated clip repositioning, ICGVA inspection of clipped IAs located at the ACoA complex seems to be especially at risk for false-negative outcomes. 3D-iDSA delivered optimal assessment of the angioarchitecture even in complex multiclip reconstructions. Our study advances the understanding first established by the few earlier comparative studies on this subject, which used portable DSA units to acquire images intraoperatively (Table 3).

Discussion about the risk and benefit of iDSA remains inconclusive. Since the introduction of ICGVA with its negligible risk of procedural complication, ${ }^{34}$ the debate has been complex, and the value of various intraoperative vessel imaging modalities remains elusive. In many studies that benchmarked ICGVA with postoperative DSA, $4,10,18,33,43$ accuracy rates were similar to those reported when comparing intraoperative and postoperative DSA. ${ }^{5,22,28,30,39}$ One could argue that ICGVA is nearly as accurate as DSA for intraoperative determination of completeness of aneurysm occlusion and parent artery patency. However, in studies in which patients undergo both ICGVA and intraoperative DSA, discordance rates between the two modalities ranged from $2 \%$ to $14 \%{ }^{4,10,18,43}$ Although this rate varied depending on the definition of "concordant results," discordance was often considered clinically significant when clip adjustment was needed. At times, neck remnants or vessel occlusion is present (and can be visualized by intraoperative DSA but not ICGVA) that cannot be corrected by clip repositioning or wrapping. In these cases, managing intraoperative and early postoperative hemodynamics can still improve patient outcomes. Accordingly, in our study limited to the detection of false-negative findings that necessitated surgical intervention, the 6\% ICGVA-iDSA discordance rate was relatively low. If all inconsistent findings between ICGVA and 3D-iDSA (including smallest dog-ear remnants) had been recorded, the discordance rate would likely have been much higher.

Another complexity in evaluating the efficacy and accuracy of ICGVA and iDSA is that no study has directly and univocally compared the two modalities. Caplan and colleagues have compared ICGVA plus iDSA with iDSA alone and reported $10.8 \%$ (18.9\% if iDSA had also detected the findings of prior ICGVA) of clip adjustments in the ICGVA-plus-iDSA group and $11.7 \%$ of clip repositioning in the iDSA-alone group. ${ }^{4}$ Accordingly ICGVA did not significantly reduce the rate of clip adjustment after routine iDSA when compared to iDSA alone (10.8\% vs $11.7 \%)$ but resulted in a remarkable risk reduction $(18.9 \%$ vs $10.8 \%)$ for clip misplacement within the combined ICGVA-plusiDSA group.

This study exemplifies the difficulty in interpreting results when the sequence and method themselves mutually influence one other. With ICGVA as an extraordinarily quick and simple intraoperative assessment, the initial clipping strategy may be less aggressive, which increases the likelihood of positive findings and thus an overestimated value of ICGVA. One could also argue that knowing iDSA will follow ICGVA may lead to a less-than-diligent interpretation of ICGVA, increase discordance, and therefore underestimate the accuracy of ICGVA. In the most comprehensive prospective comparison of various intraoperative vascular monitoring techniques in 2011, Gruber et 

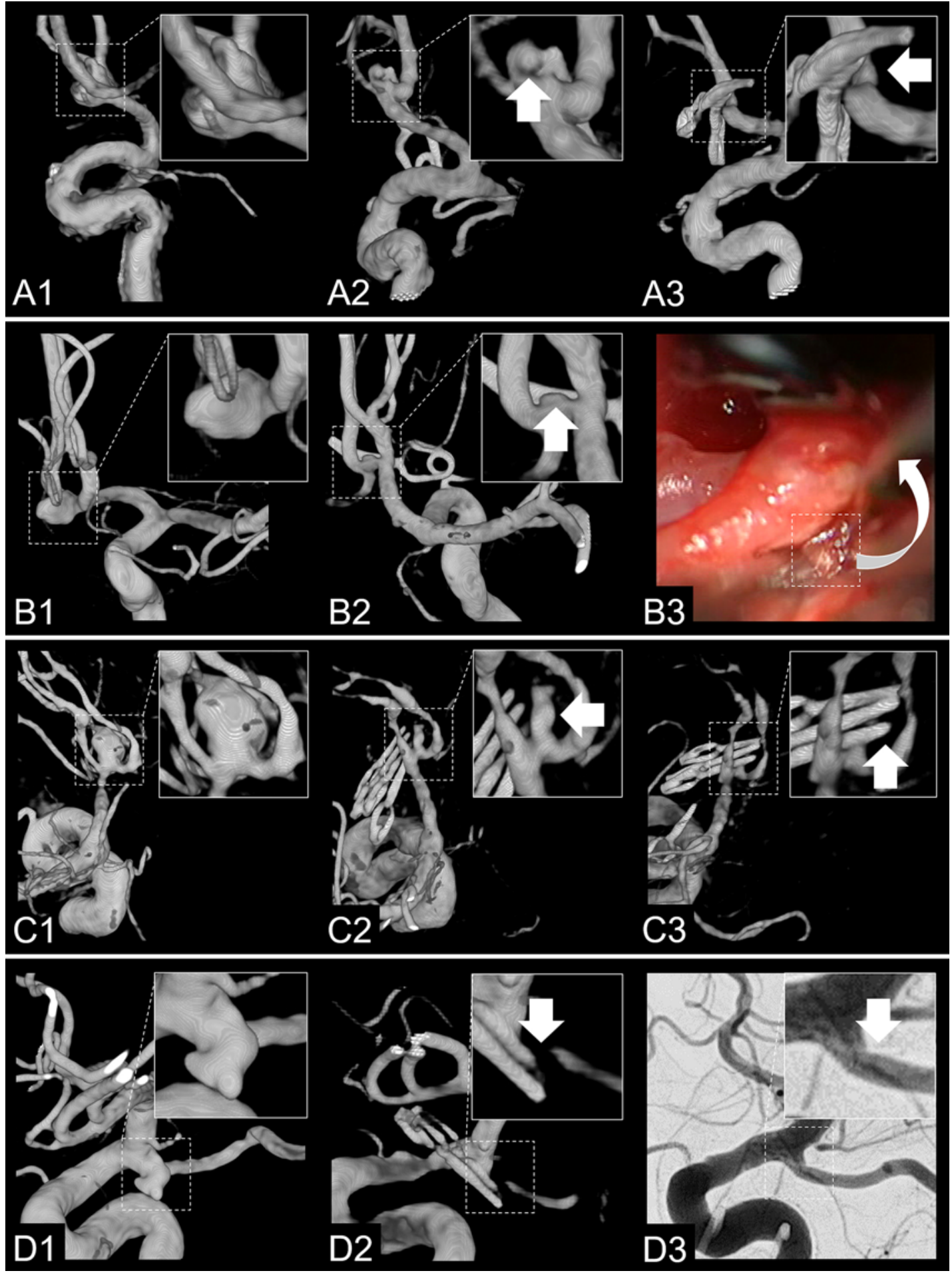

FIG. 2. Illustrative cases in which 3D-iDSA provided relevant surgical information that remained undetected after visual inspection, Doppler ultrasonography, and ICGVA. A: Aneurysm remnant treated by additional clip: 3D-iDSA revealed a residual aneurysm remnant beneath the tips of the clip blades (A2; arrow). After applying an additional curved clip, repeat 3D-iDSA demonstrated complete aneurysm obliteration (A3; arrow). B: Aneurysm remnant treated by wrapping: 3D-iDSA revealed a slight bulging beneath the middle portion of the clip (B2; arrow). Although clip repositioning was unlikely to improve the surgical result, the aneurysm remnant could be circumferentially wrapped (B3; arrow). C: Aneurysm remnant treated by clip repositioning: 3D-iDSA demonstrated significant residual aneurysm filling in the extension of the aneurysm tips (C2; arrow). Each clip was removed and repositioned more deeply to completely occlude the aneurysm (C3; arrow). D: Parent vessel impairment resolved by clip repositioning: after hemodynamically relevant stenosis was detected by 3D-iDSA (D2; arrow), the clip was repositioned, and the parent artery preserved (D3; arrow). Figure is available in color online only. 
TABLE 3. Literature comparing ICGVA to iDSA

\begin{tabular}{lcccc}
\hline \multicolumn{1}{c}{ Authors \& Year } & No. of Patients & $\begin{array}{c}\text { \% of Cohort w/ } \\
\text { ICGVA \& iDSA }\end{array}$ & $\begin{array}{c}\text { \% of Post-ICGVA } \\
\text { Clip Adjustment }\end{array}$ & $\begin{array}{c}\text { \% Correspondence } \\
\text { Btwn ICGVA \& iDSA }\end{array}$ \\
\hline Raabe et al., 2005* & 62 & $84(62 / 74)$ & $9(10 / 110)$ & $90(54 / 60)$ \\
\hline Gruber et al., 2011* & 104 & $100(104 / 104)$ & $6(7 / 123)$ & $98(120 / 123)$ \\
\hline Washington et al., 2013* & 49 & $32(49 / 155)$ & $4(2 / 49)$ & $86(42 / 49)$ \\
\hline Caplan et al., 2014* & 37 & $36(37 / 97)$ & $8(3 / 37)$ & $89(33 / 37)$ \\
\hline Defillo et al., 2014* & 230 & $98(230 / 235)$ & $6(13 / 230)$ & $96(221 / 230)$ \\
\hline & Present study† & $86(120 / 140)$ & $11(13 / 120)$ & $94 / 120)$ \\
\hline
\end{tabular}

al. included Doppler ultrasonography, ICGVA, iDSA, endoscopy, and electrophysiological monitoring. The authors concluded that the value of each tool cannot be considered separately; rather, the combination of adjuncts will perform better than the best single tool. ${ }^{18}$ Furthermore, some of the assessment methods have distinct advantages, such as the ability of ICGVA to clearly assess blood flow of visible small perforating vessels. Even the best iDSA quality lacks this resolution in the submillimeter range.,,33

While visual inspection, Doppler ultrasonography, and ICGVA are performed in a relatively straightforward manner, iDSA demands greater technical efforts. This has prompted various authors to search for predictors when iDSA would be most needed. However, the great variety of the angioarchitecture of each aneurysm makes prediction of selective use of iDSA very difficult, even in seemingly simple cases..$^{22,42}$ Prospective assessment of selective iDSA revealed that clip adjustments are needed in $4.4 \%$ of patients in whom iDSA was thought to be unnecessary. Based on the low iDSA complication rate, Klopfenstein et al. concluded that iDSA should be considered in the majority of cases. ${ }^{22}$ In a 2016 prospective study, Washington et al. confirmed that the surgeon's prediction of the necessity of intraoperative angiography is not accurate enough to justify its use on a selective basis only. ${ }^{42}$ Both preoperative and immediate postclipping assessment resulted in false-negative rates of $8 \%$ and $6 \%$, respectively. With the decreasing exposure and experience to open IA surgery, it is unlikely that the surgeon's judgment will improve. Rather, information from iDSA will gain in value.

Taken together, the frequency of false-negative findings on ICGVA, , , , 19,24,27,29,35,36,41,43 the high rate of unexpected findings on iDSA, ${ }^{5,22,28,30,39}$ and the difficulty in predicting which subgroups of patients are most likely to have surgical difficulties ${ }^{8,22,42}$ highlight the need for unselected quality control in IA surgery. Although iDSA represents the gold standard of care, $, 22,33,34,39$ its imaging quality cannot yet supplant postoperative DSA, thereby forcing clinicians to carefully balance its risk $(0.4 \%-0.99 \%$ in more recent large series ${ }^{5,39}$ ) and benefit. The inferiority of iDSA is attributed mainly to suboptimal fluoroscopic visibility and nonstandard patient positioning. ${ }^{21}$ Its false-negative findings arise from small remnants often obscured by surrounding vessels compared with the best projections of postoperative DSA. However, multiplanar 3D-DSA virtually rotates the clipped IA complex in any direction, ob- viating the need for a specific projection and minimizing interference with clip mass or adjacent vessels (Fig. 3).

Accumulating evidence suggests that 3D-DSA is superior to conventional DSA in terms of evaluating IA morphology and parent artery configuration and in detecting residual IA after clipping. ${ }^{2,20,23,38,40,44}$ When compared with 3D-DSA, Kang et al. reported that conventional DSA, even when optimized by rotation angiograms, depicted less than $50 \%$ of postoperative residual IA and $16 \%$ of dog-ear-like remnants. ${ }^{20} \mathrm{Ahn}$ and Kim reported a nearly double postclipping IA remnant detection rate of $19.3 \%$ for 3D-DSA versus $10.9 \%$ for $2 \mathrm{D}$-DSA. ${ }^{2}$ Noninvasive postoperative CTA demonstrated excellent accuracy in the evaluation of uncomplicated IAs of the anterior circulation, and it replaced routine use of 2D-DSA in many neurosurgical centers. ${ }^{6,8,11}$ Whether postoperative optimized CTA also poses a challenge to the current gold standard of 3D-DSA remains a matter of debate. ${ }^{15,16,31}$ To date, DSA is still considered the most accurate technique for assessing clipped aneurysms ${ }^{25}$ and some authors insist on routine use of postoperative 3D-DSA to validate the surgical result after aneurysm clipping. ${ }^{1}$

We have confirmed excellent imaging quality and unrestricted viewing angles of 3D-iDSA, immediately evaluating the completeness of IA occlusion and the patency of parent arteries, even in complex multiclip reconstructions. Routine intraoperative 3D visualization of the clipped IA optimized our understanding of the angioarchitecture and facilitated decision making and strategy for intraoperative improvement of the surgical result if needed. In our institution, 3D-iDSA has replaced the postoperative modality as quality control and serves as a baseline for lifelong follow-up of the clipped IA in these patients.

\section{Conclusions}

Our study confirms the great utility of both ICGVA and 3D-DSA for intraoperative assessment of the surgical result after IA clipping, corroborating earlier findings that these techniques are complementary rather than competitive. Despite its relatively low discordance rate, iDSA is the confirmed gold standard regarding surgical outcome. Improved iDSA quality in a hybrid operating room, including the capacity of 3D-iDSA to supplant postoperative DSA, supports its routine use in IA surgery. With the increased prevalence of hybrid operating rooms deliver- 


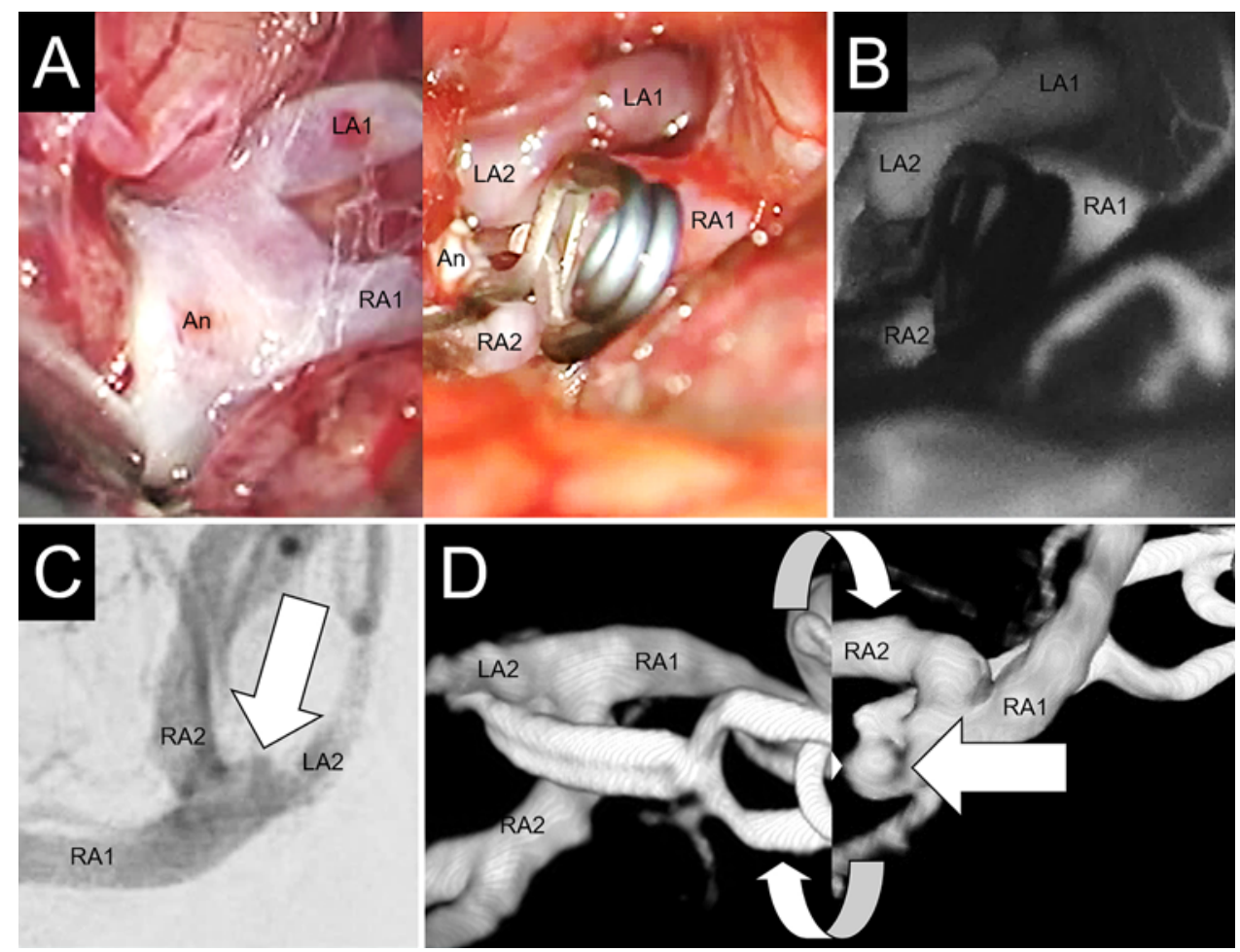

FIG. 3. Intraoperative vascular monitoring by visual inspection, ICGVA, and 2D- and 3D-iDSA. Although visual inspection before and after aneurysm clipping (A) and ICGVA (B) confirmed complete aneurysm occlusion, the best 2D-iDSA projection (C) suggested a false-negative finding (C; arrow). In this case of a deep-seated aneurysm located on the ACoA complex, the ICGVA's angle of vision limits visualization of the part of the base behind the clipped aneurysm. Multiplanar 3D-iDSA (D) completes intraoperative assessment with virtual rotation of the clipped aneurysm complex in any direction; aneurysm remnants (arrow) detected that were not visible in the surgical field. An = aneurysm; $L A 1=$ left proximal segment of $A C A ; L A 2=$ left distal segment of $A C A ; R A 1=$ right proximal segment of ACA; RA2 = right distal segment of ACA. Figure is available in color online only.

ing high-quality 3D imaging, our study serves as a base to compare the results of future studies.

\section{References}

1. Abla AA, Lawton MT: Indocyanine green angiography for cerebral aneurysm surgery: advantages, limitations, and neurosurgeon intuition. World Neurosurg 82:e585-e586, 2014

2. Ahn SS, Kim YD: Three-dimensional digital subtraction angiographic evaluation of aneurysm remnants after clip placement. J Korean Neurosurg Soc 47:185-190, 2010

3. Allcock JM, Drake CG: Postoperative angiography in cases of ruptured intracranial aneurysm. J Neurosurg 20:752-759, 1963

4. Caplan JM, Sankey E, Yang W, Radvany MG, Colby GP, Coon AL, et al: Impact of indocyanine green videoangiography on rate of clip adjustments following intraoperative angiography. Neurosurgery 75:437-444, 2014

5. Chalouhi N, Theofanis T, Jabbour P, Dumont AS, Fernando Gonzalez L, Starke RM, et al: Safety and efficacy of intraoperative angiography in craniotomies for cerebral aneurysms and arteriovenous malformations: a review of 1093 consecutive cases. Neurosurgery 71:1162-1169, 2012

6. Chen W, Yang Y, Qiu J, Peng Y, Xing W: Sixteen-row multislice computerized tomography angiography in the postoperative evaluation of patients with intracranial aneurysms. Br J Neurosurg 22:63-70, 2008

7. Chiang VL, Gailloud P, Murphy KJ, Rigamonti D, Tamargo RJ: Routine intraoperative angiography during aneurysm surgery. J Neurosurg 96:988-992, 2002
8. Dashti R, Laakso A, Niemelä M, Porras M, Hernesniemi J: Microscope-integrated near-infrared indocyanine green videoangiography during surgery of intracranial aneurysms: the Helsinki experience. Surg Neurol 71:543-550, 2009

9. de Oliveira JG, Beck J, Seifert V, Teixeira MJ, Raabe A: Assessment of flow in perforating arteries during intracranial aneurysm surgery using intraoperative near-infrared indocyanine green videoangiography. Neurosurgery 62 (6 Suppl 3):1300-1310, 2008

10. Defillo A, Qureshi M, Nussbaum ES: Indocyanine green videoangiography and intraoperative catheter digital subtraction angiography in the treatment of intracranial aneurysms: a consecutive series of 235 cases. Cureus 6:e203, 2014

11. Dehdashti AR, Binaghi S, Uske A, Regli L: Comparison of multislice computerized tomography angiography and digital subtraction angiography in the postoperative evaluation of patients with clipped aneurysms. J Neurosurg 104:395-403, 2006

12. Drake CG, Allcock JM: Postoperative angiography and the "slipped" clip. J Neurosurg 39:683-689, 1973

13. Drake CG, Vanderlinden RG: The late consequences of incomplete surgical treatment of cerebral aneurysms. J Neurosurg 27:226-238, 1967

14. Fandino J, Taussky P, Marbacher S, Muroi C, Diepers M, Fathi AR, et al: The concept of a hybrid operating room: applications in cerebrovascular surgery. Acta Neurochir Suppl 115:113-117, 2013

15. Gölitz P, Struffert T, Ganslandt O, Lang S, Knossalla F, Doerfler A: Contrast-enhanced angiographic computed tomography for detection of aneurysm remnants after clipping: 
a comparison with digital subtraction angiography in 112 clipped aneurysms. Neurosurgery 74:606-614, 2014

16. Gölitz P, Struffert T, Ganslandt O, Saake M, Lücking H, Rösch J, et al: Optimized angiographic computed tomography with intravenous contrast injection: an alternative to conventional angiography in the follow-up of clipped aneurysms? J Neurosurg 117:29-36, 2012

17. Grossart KW, Turner JW: Per-operative angiography in cerebral vascular surgery. Clin Radiol 25:279-292, 1974

18. Gruber A, Dorfer C, Standhardt H, Bavinzski G, Knosp E: Prospective comparison of intraoperative vascular monitoring technologies during cerebral aneurysm surgery. Neurosurgery 68:657-673, 2011

19. Imizu S, Kato Y, Sangli A, Oguri D, Sano H: Assessment of incomplete clipping of aneurysms intraoperatively by a nearinfrared indocyanine green-video angiography (Niicg-Va) integrated microscope. Minim Invasive Neurosurg 51:199_ 203,2008

20. Kang HS, Han MH, Kwon BJ, Jung SI, Oh CW, Han DH, et al: Postoperative 3D angiography in intracranial aneurysms. AJNR Am J Neuroradiol 25:1463-1469, 2004

21. Katz JM, Gologorsky Y, Tsiouris AJ, Wells-Roth D, Mascitelli J, Gobin YP, et al: Is routine intraoperative angiography in the surgical treatment of cerebral aneurysms justified? A consecutive series of 147 aneurysms. Neurosurgery 58:719727,2006

22. Klopfenstein JD, Spetzler RF, Kim LJ, Feiz-Erfan I, Han PP, Zabramski JM, et al: Comparison of routine and selective use of intraoperative angiography during aneurysm surgery: a prospective assessment. J Neurosurg 100:230-235, 2004

23. Kumar S, Gaikwad SB, Mishra NK: 3D rotational angiography in follow-up of clipped intracranial aneurysms. ISRN Radiol 2014:935280, 2014

24. Li J, Lan Z, He M, You C: Assessment of microscope-integrated indocyanine green angiography during intracranial aneurysm surgery: a retrospective study of 120 patients. Neurol India 57:453-459, 2009

25. Lin A, Rawal S, Agid R, Mandell DM: Cerebrovascular imaging: which test is best? Neurosurgery [epub ahead of print], 2017

26. Marbacher S, Diepers M, Kahles T, Nedeltchev K, Remonda $\mathrm{L}$, Fandino J: Interdisciplinary decision-making and treatment of intracranial aneurysms in the era of complementary microsurgical and endovascular techniques. Swiss Med Wkly 146:w14372, 2016

27. Moon HS, Joo SP, Seo BR, Jang JW, Kim JH, Kim TS: Value of indocyanine green videoangiography in deciding the completeness of cerebrovascular surgery. J Korean Neurosurg Soc 53:349-355, 2013

28. Origitano TC, Schwartz K, Anderson D, Azar-Kia B, Reichman OH: Optimal clip application and intraoperative angiography for intracranial aneurysms. Surg Neurol 51:117-128, 1999

29. Özgiray E, Aktüre E, Patel N, Baggott C, Bozkurt M, Niemann D, et al: How reliable and accurate is indocyanine green video angiography in the evaluation of aneurysm obliteration? Clin Neurol Neurosurg 115:870-878, 2013

30. Payner TD, Horner TG, Leipzig TJ, Scott JA, Gilmor RL, DeNardo AJ: Role of intraoperative angiography in the surgical treatment of cerebral aneurysms. J Neurosurg 88:441-448, 1998

31. Psychogios MN, Wachter D, Mohr A, Schramm P, Frölich AM, Jung K, et al: Feasibility of flat panel angiographic CT after intravenous contrast agent application in the postoperative evaluation of patients with clipped aneurysms. AJNR Am J Neuroradiol 32:1956-1962, 2011

32. Raabe A, Beck J, Gerlach R, Zimmermann M, Seifert V: Near-infrared indocyanine green video angiography: a new method for intraoperative assessment of vascular flow. Neurosurgery 52:132-139, 2003
33. Raabe A, Nakaji P, Beck J, Kim LJ, Hsu FP, Kamerman JD, et al: Prospective evaluation of surgical microscopeintegrated intraoperative near-infrared indocyanine green videoangiography during aneurysm surgery. J Neurosurg 103:982-989, 2005

34. Riva M, Amin-Hanjani S, Giussani C, De Witte O, Bruneau $\mathrm{M}$ : Indocyanine green videoangiography in aneurysm surgery: systematic review and meta-analysis. Neurosurgery [epub ahead of print], 2017

35. Roessler K, Krawagna M, Dörfler A, Buchfelder M, Ganslandt O: Essentials in intraoperative indocyanine green videoangiography assessment for intracranial aneurysm surgery: conclusions from 295 consecutively clipped aneurysms and review of the literature. Neurosurg Focus 36(2):E7, 2014

36. Sharma M, Ambekar S, Ahmed O, Nixon M, Sharma A, Nanda A, et al: The utility and limitations of intraoperative near-infrared indocyanine green videoangiography in aneurysm surgery. World Neurosurg 82:e607-e613, 2014

37. Steven JL: Postoperative angiography in treatment of intracranial aneurysms. Acta Radiol Diagn (Stockh) 5:536-548, 1966

38. Sugahara T, Korogi Y, Nakashima K, Hamatake S, Honda S, Takahashi M: Comparison of 2D and 3D digital subtraction angiography in evaluation of intracranial aneurysms. AJNR Am J Neuroradiol 23:1545-1552, 2002

39. Tang G, Cawley CM, Dion JE, Barrow DL: Intraoperative angiography during aneurysm surgery: a prospective evaluation of efficacy. J Neurosurg 96:993-999, 2002

40. van Rooij WJ, Sprengers ME, de Gast AN, Peluso JP, Sluzewski M: 3D rotational angiography: the new gold standard in the detection of additional intracranial aneurysms. AJNR Am J Neuroradiol 29:976-979, 2008

41. Wang S, Liu L, Zhao Y, Zhang D, Yang M, Zhao J: Evaluation of surgical microscope-integrated intraoperative near-infrared indocyanine green videoangiography during aneurysm surgery. Neurosurg Rev 34:209-215, 2010

42. Washington CW, Derdeyn CP, Chicoine MR, Cross DT, Dacey RG, Moran CJ, et al: Comparing routine versus selective use of intraoperative cerebral angiography in aneurysm surgery: a prospective study. J Neurointerv Surg 8:75-80, 2016

43. Washington CW, Zipfel GJ, Chicoine MR, Derdeyn CP, Rich $\mathrm{KM}$, Moran CJ, et al: Comparing indocyanine green videoangiography to the gold standard of intraoperative digital subtraction angiography used in aneurysm surgery. J Neurosurg 118:420-427, 2013

44. Wong SC, Nawawi O, Ramli N, Abd Kadir KA: Benefits of 3D rotational DSA compared with 2D DSA in the evaluation of intracranial aneurysm. Acad Radiol 19:701-707, 2012

\section{Disclosures}

The authors report no conflict of interest concerning the materials or methods used in this study or the findings specified in this paper.

\section{Author Contributions}

Conception and design: Marbacher, Fandino. Acquisition of data: Mendelowitsch. Analysis and interpretation of data: Marbacher, Mendelowitsch, Grüter. Drafting the article: Marbacher, Mendelowitsch, Diepers. Critically revising the article: Marbacher, Fandino. Reviewed submitted version of manuscript: Marbacher, Grüter, Diepers, Remonda, Fandino. Statistical analysis: Marbacher. Administrative/technical/material support: Remonda, Fandino. Study supervision: Fandino.

\section{Correspondence}

Itai Mendelowitsch: Kantonsspital Aarau, Switzerland. itai. mendelowitsch@ksa.ch. 